\title{
Is left ventricular hypertrophy a friend or foe of patients with aortic stenosis?
}

\author{
Cecilia Davies ${ }^{1}$, Katherine Zerebiec ${ }^{1}$, Agnieszka Rożanowska ${ }^{1}$, Ewa Czestkowska ${ }^{1}$, Dorota Długosz ${ }^{1}$, \\ Bernadeta Chyrchel ${ }^{2}$, Andrzej Surdacki ${ }^{2}$ \\ 'Students' Scientific Group at the Second Department of Cardiology, Jagiellonian University Medical College, Krakow, Poland \\ ${ }^{2}$ Second Department of Cardiology, Jagiellonian University Medical College, Krakow, Poland
}

Adv Interv Cardiol 2018; 14, 4 (54): 328-337

DOI: https://doi.org/10.5114/aic.2018.78734

\begin{abstract}
Left ventricular hypertrophy (LVH) is traditionally considered a physiological compensatory response to LV pressure overload, such as hypertension and aortic stenosis (AS), in an effort to maintain LV systolic function in the face of an increased afterload. According to the Laplace law, LV wall thickening lowers LV wall stress, which in turn would be helpful to preserve LV systolic performance. However, numerous studies have challenged the notion of LVH as a putative beneficial adaptive mechanism. In fact, the magnitude of LVH is associated with higher cardiovascular morbidity and mortality, especially when LVH is disproportionate to LV afterload. We have briefly reviewed: first, the importance of non-valvular factors, beyond AS severity, for total LV afterload and symptomatic status in AS patients; second, associations of excessive LVH with LV dysfunction and adverse outcome in AS; third, prognostic relevance of the presence or absence of pre-operative LVH in patients referred for aortic valve surgery; fourth, time course, determinants and prognostic implications of LVH regression and LV function recovery after surgical valve replacement and transcatheter aortic valve implantation (TAVI) with a focus on TAVI-specific effects; fifth, the potential of medical therapy to modulate LVH before and after surgical or interventional treatment for severe AS, a condition perceived as a relative contraindication to renin-angiotensin system blockade.
\end{abstract}

Key words: aortic valve stenosis, cardiac valve surgery, left ventricular function, left ventricular hypertrophy, prognosis, transcatheter aortic valve implantation.

\section{Introduction}

Every medical student is taught that the compensatory response to increased left ventricular (LV) afterload by the heart is left ventricular hypertrophy (LVH). The heart muscle increases the size of myocytes, making the heart stronger and better able to cope with the increased pressure it has to pump against.

A common cause of increased LV afterload on the heart, especially when we age, is the age-dependent increase in blood pressure, frequently associated with reduced compliance of large systemic arteries, a basis of isolated systolic hypertension [1]. Degenerative changes of aortic valve leaflets sometimes progress into aortic valve stenosis (AS), the most prevalent valve disease in the elderly, which further contributes to LV pressure overload. Then, both hypertension and AS jointly impede blood flow from the left ventricle into the aorta and enhance LVH development.
According to traditional teaching, $\mathrm{LVH}$ is perceived as a compensatory - and probably also beneficial - mechanism aimed to lower LV wall stress by the Laplace law, thereby facilitating LV systolic performance despite chronic pressure overload. However, the notion of LVH as an advantageous LV adaptation has been challenged by recent evidence, suggesting that perhaps we have been thinking about LVH incorrectly. In essential hypertension, it has been demonstrated that the degree of LVH is associated with adverse outcomes [2], especially when the magnitude of LVH is disproportionate to LV afterload. Excessive LVH is associated with LV diastolic [3] and subtle systolic dysfunction [3-5] as well as impaired coronary flow reserve [6] in hypertensive subjects, which accelerates the development of heart failure and translates into worse long-term prognosis [7, 8].

We aimed to review: first, the importance of non-valvular factors, beyond AS severity, for total LV afterload and symptomatic status in AS patients; second, associ-

\section{Corresponding author:}

Andrzej Surdacki, Second Department of Cardiology, Jagiellonian University Medical College, 17 Kopernika St, 31-501 Krakow, Poland, phone/fax: +48 1242471 80, e-mail: surdacki.andreas@gmx.net

Received: 28.07.2018, accepted: 15.08.2018. 
ations of excessive LVH with LV dysfunction and adverse outcome in AS; third, prognostic relevance of the presence or absence of pre-operative LVH in patients referred for aortic valve surgery; fourth, time course, determinants and prognostic implications of LVH regression and LV function recovery after surgical valve replacement and transcatheter aortic valve implantation (TAVI) with a focus on TAVI-specific effects; fifth, the potential of medical therapy to modulate LVH before and after surgical or interventional treatment for severe AS, a condition perceived as a relative contraindication to renin-angiotensin system blockade.

\section{Vascular stiffening - a contributor to LV afterload and dysfunction in AS}

Depressed compliance of large systemic arteries, i.e. reduced systemic arterial compliance (SAC), is observed during normal human aging. Beyond age, diabetes, renal disease, hypertension and atherosclerosis all contribute to decreased arterial distensibility and compliance [1,9]. These abnormalities are linked to progressive fragmentation of elastic fibers and increased collagen content in the thickened vascular wall, as well as excessive cross-linking of elastin and collagen, which transfers the mechanical load into much stiffer collagen fibers. Accordingly, the vessel becomes less distensible and as a result the pulse wave velocity (PWV) increases. Pulse wave velocity is commonly understood as the speed at which the pressure wave from the heart moves through the vascular system, and its measurement has become the gold standard for measuring SAC. When the PWV is greater, as it occurs in non-compliant elastic arteries, there is a phenomenon of increased late systolic pressure due to an earlier return of the pulse wave reflection back to the heart. This causes a higher central systolic pressure in the elderly which ultimately leads to increases in the LV afterload [9].

The increased afterload from large vessel stiffening consequentially slows LV relaxation, which corresponds to an early phase of diastolic dysfunction. A concomitant reduction in central diastolic pressure may also compromise coronary perfusion and potentiate subendocardial ischemia, which can further impair myocardial relaxation and promote myocardial fibrosis, aggravating diastolic dysfunction via increased passive myocardial stiffness [9]. This destructive cycle initiates the development of heart failure symptoms in AS, despite a normal ejection fraction (EF), and provides a link between depressed SAC and heart failure with preserved EF.

Briand et al. [10] emphasized that reduced SAC was a critical component in the pathogenesis of LV dysfunction, acting via increased LV afterload. They demonstrated that in patients with AS accompanied by severely depressed SAC and consequent systolic hypertension, valvulo-arterial impedance, an index of global LV after- load, can be especially high, which predisposes them to the occurrence of both symptoms and LV systolic and diastolic dysfunction independently of AS severity [10]. They argued that assessing AS based solely on valvular hemodynamics can overlook contributing factors distal to the valve that are responsible for elevated afterload. In particular, developing LV systolic dysfunction may result in stroke volume lowering and systolic pressure pseudo-normalization, which can mask the contribution of excessive arterial stiffness to LV afterload. Accordingly, they recommended routine calculations of SAC from echocardiography - as stroke volume index divided by pulse pressure - to avoid this pitfall [10]. This further demonstrates that the degenerative process in largely elderly patients with AS is a systemic problem that is not confined to the aortic valve.

\section{Non-valvular factors - predictors of symptoms development in AS}

As mentioned above, depressed SAC and elevated valvulo-arterial impedance are associated not only with LV systolic and diastolic dysfunction, but also symptomatic status in AS [10]. This notion has been widely supported by later evidence with regard to exercise capacity in AS subjects. In patients with asymptomatic moderate or severe AS and a preserved EF, increased arterial valvulo-arterial impedance was an independent predictor of reduced peak oxygen uptake, as shown by Dulgheru et al. [11]. Similarly, the vascular component of LV afterload in AS appears to be associated with the presence of symptoms [12] or exercise intolerance [13]. In agreement with these observations, our group has recently shown that depressed SAC predicted the degree of exercise intolerance independently of AS severity and EF, in a retrospective cross-sectional analysis of patients with moderate-to-severe AS [14], which supports the relevance of non-valvular factors for symptomatic status.

Thus, vascular factors supplement a set of independent correlates of symptoms development, beyond pure AS severity and EF, including subtle LV systolic impairment [15], diastolic dysfunction [16] and elevated LV filling pressures [17]. Because the risk of mortality rises dramatically after the onset of symptoms in AS, exploration of mechanisms underlying early changes in LV structure appears crucial for better understanding of AS natural history. Since LVH frequently develops in an early stage of AS and precedes symptoms development, prognostic consequences of LVH has long attracted researchers' attention.

\section{Left ventricular hypertrophy - a predictor of adverse outcome in AS}

According to traditional teaching, LVH reflects LV adaptation to pressure overload and enables the maintenance of LV systolic performance in AS. However, contrary 
to expectations, the magnitude of LVH is associated with worse, not better, prognosis. In a prospective study, Gerdts et al. [18] evaluated LV mass and its prognostic significance in 1,656 patients with asymptomatic mild-moderate AS with no evidence of coronary heart disease, heart failure, peripheral artery disease, diabetes or renal insufficiency, participating in the SEAS trial. By following the patients for about 4 years, they were able to demonstrate that a higher LV mass at baseline was associated with various clinical end-point outcomes, i.e. all-cause death, cardiovascular (CV) death, ischemic CV events (myocardial infarction, stroke, coronary revascularization and CV death) and so-called aortic valve events, i.e. a joint composite outcome of aortic valve replacement, CV death or hospitalization for heart failure due to AS [18]. It is noteworthy that these associations, when quantified per 1-SD increase in LV mass, were stronger for LV mass during the follow-up ("in-study" LV mass) compared to baseline LV mass [18]. Additionally, the negative prognostic effect of in-study LV mass, when analyzed as a time-dependent covariate, remained significant upon extensive multivariate adjustment for AS severity, age, sex, body mass index, hypertension, randomized study treatment, EF, LV concentricity (i.e. relative LV wall thickness) and valvulo-arterial impedance [18]. Accordingly, the ability of LV mass to predict adverse CV events appears to be linked to LVH by itself, not to a combination of LVH and risk factors affecting outcome. In particular, during the 4-year period, the annual progression rate of AS severity was similar in the LVH and non-LVH group, despite LVH prevalence nearly doubling [18]. Even though LVH has largely been considered a compensatory response to increased systolic LV pressures from AS, Gerdts et al. suggested that LVH development appears multifactorial with its detrimental effects prevailing, especially when LV mass is excessive relative to hemodynamic load [18].

The concept of excessive LVH was first suggested by Italian researchers who validated a formula which enables the quantification of inappropriately high LV mass, i.e. beyond the value predicted from hemodynamic load, gender and height $[4,19]$. In their hands, excessive LVH was defined as an observed-to-predicted LV mass ratio greater than $128 \%$, i.e. the $95^{\text {th }}$ percentile of its distribution in a reference population of 393 normotensive subjects, and termed "inappropriately high LV mass (iLVM)" $[4,19]$. The prevalence of iLVM was as high as $37 \%$ in 941 hypertensive patients enrolled in the LIFE study [20], whereas the respective proportion averaged $16.6 \%$ in 1,614 SEAS trial participants with mild or moderate AS [21], and $24 \%$ in an early report by Mureddu et al. [22] of 100 AS subjects. Of note, in the latter study, the prevalence of iLVM tended to increase with the degree of AS severity, averaging $16 \%, 26 \%$ and $32 \%$ in those with a mild, moderate or severe grade of valve obstruction, respectively [22]. Like in essential hypertension [20], meta- bolic risk factors (obesity and metabolic syndrome) were also confirmed as "non-hemodynamic" contributors to iLVM in AS subjects with mild or moderate AS recruited to the SEAS [23] and ASTRONOMER [24] trials.

Cioffi et al. [21] not only reported the prevalence of iLVM in AS, but also described its correlates. According to their study, compared to patients with an appropriate $\mathrm{LV}$ mass (i.e., between $73 \%$ and $128 \%$ of the measured-to-predicted LV mass), subjects with iLVM did not differ in age, AS severity or prevalence or severity of hypertension. In contrast, those with iLVM were more frequently male, with a considerably higher relative LV wall thickness (by 35\%). Of note, iLVM was associated with decreased circumferential end-systolic LV wall stress (by $10 \%$ ), slightly lower EF (by $4 \%$ ) but pronounced (by $30 \%)$ reductions of stress-corrected LV midwall fractional shortening [21], a parameter better than EF as an index of LV systolic performance in concentric LV geometry [25]. Importantly, as many as $88 \%$ of patients with iLVM had depressed stress-corrected midwall fractional shortening, compared to only $15 \%$ in those with an appropriate LV mass [21].

Based on these findings, Cioffi et al. [21] concluded that iLVM in asymptomatic AS was probably a sign of concomitant load-independent primary myocardial dysfunction which could underlie excessive LVH as an ineffective compensatory mechanism whose goal would be to restore LV performance via reduced systolic LV wall stress in accordance with the Laplace law. That LV end-diastolic internal diameter, a raw index of preload, was only slightly lower (by $3 \%$ ) in the presence of iLVM is also in agreement with this hypothesis [21]. The notion of depressed LV contractility offers a plausible explanation for the association of iLVM and impaired LV midwall systolic function with a surprisingly lower, not higher, LV afterload [21], consistent with analogous observations in hypertensive patients $[5,26]$.

Development of excessive LVH appears not only a correlate of intrinsic LV systolic dysfunction [3-5, 21], but is also associated with impaired coronary flow reserve [6] and LV diastolic dysfunction [3], a substrate of heart failure with preserved EF [8], the predominant form of heart failure in the elderly. These abnormalities may be responsible for the independent ability of iLVM, but not LVH or LV mass index, to predict adverse CV outcome in 209 patients with asymptomatic severe AS free from coexistent diseases (except for hypertension and diabetes) followed for almost 2 years [27]. Although iLVM was associated with depressed stress-corrected LV midwall fractional shortening, only iLVM entered the final regression model as an independent prognosticator [27]. The results were similar after exclusion of aortic valve replacement from the composite end-point outcome encompassing also all-cause death and hospital admissions for myocardial infarction or heart failure. That patients with iLVM had a 4.5-higher risk of any of these adverse CV events than 
their counterparts with appropriate LVH [27] strongly supports the clinical relevance of excessive LVH in AS.

\section{Prognostic implications of the presence or absence of pre-operative LVH in AS patients undergoing surgical aortic valve replacement}

Early reports clearly defined associations between pre-operative LVH and mortality after surgical aortic valve replacement. In a group of 60 AS subjects, Orsinelli et al. [28] identified higher relative LV wall thickness, an index of LV concentricity, as a risk factor for early in-hospital mortality following surgery. In a large survey of 5,083 patients operated on for AS, Duncan et al. [29] described greater LV concentricity, not LVH by itself, as an independent predictor of in-hospital mortality. That concentric LV geometry may pose a risk for late post-operative prognosis was also suggested by Debry et al. [30], who reported that only LV concentric remodeling (i.e. elevated relative LV wall thickness in the absence of LVH) independently predicted mortality over a mean follow-up of about 3 years when surgical management was considered, in 331 asymptomatic or mildly symptomatic patients with AS and preserved EF. With regard to AS subjects under conservative management, the highest risk of 3-4-year mortality was found for concentric remodeling followed by concentric LVH [30].

Intriguingly, not only the magnitude of LVH predicts adverse early and late post-operative outcome, but also the lack of LVH, a putative compensatory mechanism, is not associated with worse prognosis. As early as over 20 years ago, Seiler and Jenni [31] observed an absence of LVH according to LV mass criteria in 21 out of 189 study subjects with severe AS and no coronary artery disease. Those LVHfree AS patients exhibited either concentric LV remodeling (57\%) or normal LV geometry (43\%) [31]. Counterintuitively, ergometric working capacity was higher in the absence of LVH. Importantly, 5-6-month survival after aortic valve replacement was not significantly worse in patients without LVH, but post-operative deaths occurred exclusively in patients with elevated relative LV wall thickness irrespective of LVH [31]. Similar findings were reported 18 years later by Barasch et al. [32], who observed no LVH in $36 \%$ of 512 subjects with severe symptomatic AS and a preserved EF. In that study, rates of aortic valve replacement were similar regardless of the presence of $\mathrm{LVH}$, and 3.5-year mortality tended to be even lower among patients without LVH [32]. Additionally, a significantly improved survival was found only in subjects with neither LVH nor concentric remodeling [32]. Accordingly, these studies further support the previously mentioned negative prognostic effect of LV concentricity [28-30]. Barasch et al. [32] speculated that as yet unidentified mechanisms are likely to offset the potential detrimental effect of excessive LV afterload in AS patients free of LVH and with a normal LV geometry.
The above unexpected findings in AS subjects without LVH may reflect pathophysiological mechanisms which are not limited to AS, being operational also in hypertension, the most prevalent cause of LV pressure overload. Indeed, patients with inadequate LV mass, i.e. the measured LV mass below the $10^{\text {th }}$ [27] or the $2.5^{\text {th }}$ percentile [33] of the distribution of the LV mass predicted from the individual hemodynamic load in the reference population [4, 19], had a similar rate of adverse CV events to those with appropriate LV mass in 209 patients with asymptomatic severe AS [27] and 294 hypertensive subjects [33]. Intriguingly, in the latter study, inadequate LV mass was associated with supranormal afterload-corrected midwall and endocardial systolic function, which, jointly with excessive afterload, elevated cardiac index and marginally higher heart rate, was suggestive of sympathetic activation [33].

Detailed pathways underlying the protective role of absent LVH despite afterload excess and possible sympathetic predominance remain to be elucidated and may also be applicable to patients with AS.

\section{Relevance of gender for $\mathrm{LV}$ remodeling in AS}

Sex-dependent differences in LV remodeling were reported in patients with AS. Compared to men, women with severe isolated AS exhibit a smaller LV size with greater LV concentricity and higher endocardial fractional shortening and EF [34]. These differences were linked to enhanced expression of profibrotic genes and myocardial fibrosis in men, presenting frequently with more eccentric LVH associated with LV dilation [35]. It was also suggested that a lower magnitude of LV fibrosis could also be responsible for faster regression of LVF after aortic valve replacement in females [36].

On the basis of their previous findings [36], in an elegant study Petrov et al. [37] calculated the so-called LVH index, a single parameter which integrates LV concentricity and LVH appropriateness relative to LV afterload. More negative values of the LVH index correspond to more appropriate afterload-corrected LV mass and concentric LV geometry, i.e. adaptive LVH, while positive values reflect maladaptive LV remodeling with a larger LV cavity and excessively high LV mass disproportionate to LV afterload [37]. The authors demonstrated a higher proportion of maladaptive LV remodeling (i.e. a positive LVH index) in men versus women, especially about 3-4 years after aortic valve replacement for severe AS ( $51 \%$ vs. $25 \%$ ), but also pre-operatively ( $55 \%$ vs. $38 \%$ for males and females, respectively) [37]. Moreover, maladaptive LV remodeling at surgery was associated with worse 3-year survival exclusively in women. Recently, the presence of residual LVH after aortic valve surgery was linked to 6-year incidence of non-fatal CV hospitalizations especially in wom- 
en [38], which further supports the long-term prognostic significance of gender after aortic valve implantation.

\section{Left ventricular hypertrophy regression following surgical aortic valve replacement - prognostic implications}

Aortic valve replacement decreases LV afterload, counteracting the main stimulus for LVH in AS. However, although LVH regression is a well-recognized phenomenon following surgery, clinical consequences of post-operative LV mass lowering are far from being unequivocally established. Obviously, although LVH regression occurs rather uniformly irrespective of valve type, predominantly during the first 3-6 months after surgery, EF improvement is evident only in patients with pre-operative LV dysfunction [39]. Several pivotal studies identified the time course, determinants and prognostic consequences of $\mathrm{LVH}$ regression after surgical aortic valve replacement for severe AS. Ali et al. [40] demonstrated that only LV mass reduction of more than $150 \mathrm{~g}$ at 1 year after surgery was an independent, albeit rather weak, predictor of improved 10-year actuarial survival. Later studies of Beach et al. [41] and Hatani et al. [42] demonstrated a regression of $\mathrm{LVH}$ during the first months after surgery, whereas LV mass remained relatively constant after 1-2 years. This is in agreement with a report of a large series of AS subjects with pre-operative LVH, described by Une et al. [43], who experienced maximal LV mass regression at a mean of 25 months.

In contrast, left atrial (LA) diameter decreased rapidly during the first month in selected individuals and then reached a plateau [42], suggesting the contribution of acute LV unloading to the normalization of LA volume. Beach et al. [41] observed associations of residual post-operative $\mathrm{LVH}$ at 2 years with - in decreasing level of importance - pre-operative LVH, larger pre-procedural LA diameter, and worse LV systolic function. Hatani et al. [42] reported independent relations between post-operative LVH at 1 year and pre-operative LVH and LA dilatation, while pre-procedural LA dilatation was the strongest correlate of post-procedural LA enlargement in both studies [41, 42]. Although residual LVH at 2 years was a univariate predictor of late all-cause mortality at 5-10 years, as reported by Beach et al. [41], severe pre-operative $\mathrm{LVH}\left(\geq 180 \mathrm{~g} / \mathrm{m}^{2}\right)$ was a more significant prognosticator, albeit weaker than severe LA dilatation $(\geq 5 \mathrm{~cm})$, because post-operative and pre-operative LV mass were strongly interrelated. They concluded that marked LVH and LA dilatation corresponded to reduced long-term survival in even asymptomatic AS patients and early surgical intervention could possibly be beneficial in these subjects [41]. In partial accordance with these findings, Hatani et al. [42] observed a higher 3-year risk of either total mortality or adverse CV events in those with both residual post-operative LVH (according to the classical criteria) and persistent LA enlargement $\left(\geq 40 \mathrm{ml} / \mathrm{m}^{2}\right)$ at 1 year after successful aortic valve replacement, compared to the remainder.

Thus, independent contributions of LVH and LA size, a marker of increased LV filling pressure, to long-term prognosis after aortic valve replacement indicate the relevance of both pre-procedural LV diastolic dysfunction and its substrate, i.e. LVH, especially in AS subjects with persistent $\mathrm{LVH}$ at $1-2$ years after surgery.

\section{Left ventricular hypertrophy regression following transcatheter aortic valve implantation - prognostic implications}

Like after aortic valve surgery, LVH regression takes place also after TAVI. However, there are considerable differences in LV remodeling after TAVI compared to surgical aortic valve replacement. The large data set of the PARTNER trial allowed the time course of LV mass changes and its determinants in patients referred for TAVI to be studied in detail. In addition, comparisons could be made between subjects undergoing TAVI and surgical aortic valve replacement [44].

In a longitudinal echocardiographic study of highrisk patients enrolled in the PARTNER I trial (cohort A), LV mass index regression was slower in TAVI patients versus those randomized to surgery (TAVI vs. surgery: $0.7 \%$ vs. $6.7 \%$ at 1 months), but the magnitude of LVH regression at 1-2 years was similar (TAVI vs. surgery: $9.4 \%$ vs. $13.3 \%$ at 1 year; $17.3 \%$ vs. $21.9 \%$ at 2 years) [45]. It was proposed that this effect could be linked to higher occurrence of aortic regurgitation caused by post-TAVI paravalvular leak (PVL), whose degree diminished over time (TAVI vs. surgery: $12.2 \%$ vs. $0.9 \%$ at 1 month and $6.8 \%$ vs. $1.9 \%$ at 1 year for moderate to severe PVL) [44]. So, post-TAVI PVL could first delay LVH regression, which might later catch up with LV remodeling recovery in the surgical cohort owing to a gradual decrease of PVL magnitude $[45,46]$.

It is noteworthy that improvements in EF were independent of $\mathrm{LVH}$ regression in the PARTNER cohort $\mathrm{A}$. Despite delayed $\mathrm{LVH}$ regression in TAVI patients, EF increased already at discharge (by about 6\%), whereas after surgery a similar change in EF was found not earlier than at 1 month, yet with similar improvements in EF (by $8 \%$ vs. baseline) in both groups at $1-2$ years [45]. Importantly, post-TAVI rises in EF (by $6 \%$ and $7 \%$ at 1 month and 1 year, respectively) preceded LVH regression also in inoperable extreme-risk patients from the PARTNER cohort B, in whom the LV mass index fell by $14 \%$ at 1 year (similar to cohort A), but by less than $3 \%$ at 1 month [47].

In contrast to PVL, the prevalence of prosthesis-patient mismatch (PPM), diagnosed when the effective orifice area is too small for the patient's body size $\left(\leq 0.85 \mathrm{~cm}^{2} /\right.$ $\mathrm{m}^{2} ;<0.65 \mathrm{~cm}^{2} / \mathrm{m}^{2}$ for severe PPM) was higher in surgically managed AS patients (surgery vs. TAVI: $60.0 \%$ vs. 
43.8-46.4\%, or $28.1 \%$ vs. $13.6-19.7 \%$ ) [48]. In multivariate analysis, any PPM was associated with less LVH regression at 1 year in the surgical arm of the PARTNER cohort $A$ and the TAVI nonrandomized continued access registry cohort [48]. Severe PPM independently predicted higher 2-year mortality in the randomized surgical cohort and 1-year mortality in the TAVI registry cohort without post-procedural aortic regurgitation [48].

Among patients with severe LVH $\left(\geq 149 \mathrm{~g} / \mathrm{m}^{2}\right.$ in men; $\geq 122 \mathrm{~g} / \mathrm{m}^{2}$ in women) included in the PARTNER cohort $A$ and the nonrandomized registry who survived 1 year after TAVI, 2 patterns of LVH regression were identified [49]. In about one-half of the subjects, an early decline of LV mass occurred at 1 month, with no further changes during the remainder of the year. As this was paralleled by a drop in relative LV wall thickness on the 30-day echocardiogram, LVH regression resulted primarily from rapid decreases in LV wall thickness, not altered LV dimensions. In the remainder, LV mass decreased gradually between 1 month and 1 year, although final 1 -year relative LV mass reductions were lower (12\%) than in those with greater LV mass regression at 1 month (23\%) [49]. Early LVH regression was independently predicted by female gender, absence of a pacemaker at baseline, greater pre-procedural LV midwall shortening and lower mean transvalvular pressure gradient at 1 month after TAVI, not PPM or aortic regurgitation [49]. Notably, the pattern of rapid regression of severe LVH was associated with a 2-fold lower rate of hospitalizations, mainly for heart failure, at 1-year follow-up, and a trend toward better quality of life [49].

The relationship between female sex and earlier/ greater post-TAVI LVH regression [49] could have contributed, in addition to higher baseline comorbidity among men [44, 50], to a lower (by about one-third) risk of allcause 2-year mortality in women, randomized to TAVI, especially by a transfemoral approach, from the PARTNER cohort A [51].

Beneficial effects of LV mass regression could also be responsible for an association of pre-operative concentric LV remodeling with higher all-cause mortality at 1 year after TAVI, as recently reported by Rymuza et al. [52]. Indeed, LV mass tended to increase after TAVI only in those with concentric remodeling, in contrast to opposite trends in those with concentric or eccentric LVH [52].

\section{Transcatheter aortic valve implantation- -specific effects on LV function recovery}

A variety of mechanisms have been proposed as a basis for clinical benefits of LVH regression after TAVI. Although Tzikas et al. [53] did not observe any improvement of LV function despite post-TAVI LVH regression 1 year after TAVI, later reports suggested better LV function in post-TAVI subjects vs. surgery. First, as previously mentioned, TAVI was associated with earlier EF improvements than surgery, preceding LVH regression [45]. Second, in a tissue tracking cardiac magnetic resonance study, Nucifora et al. [54] observed correlations between the degree of LV mass regression and improvement in global LV strain in 3 directions (longitudinal, radial and circumferential) at about 15 months after both TAVI and surgical aortic valve implantation. Moreover, an early post-procedural increase in global LV longitudinal strain was found only after TAVI, in contrast to a transient decrease in that parameter after surgery, despite no changes of EF [46]. Thus, compared to surgery, TAVI might be associated with an additional early benefit for LV systolic function, regardless of that accompanying late $\mathrm{LVH}$ regression. Third, Costantino et al. [55] reported greater decreases in LV mass, relative wall thickness and the E/E' ratio, a noninvasive estimate of LV filling pressure in post-TAVI versus surgical patients at 2 months after the procedure. Furthermore, 2-month RWT reductions and simultaneous lowering of the $E / E^{\prime}$ ratio were interrelated exclusively after TAVI, not after surgery, regardless of post-procedural falls in valvulo-arterial impedance [55].

\section{Renin-angiotensin system blockade in AS - a tool to inhibit LVH development and potentiate post-procedural LVH regression?}

Clinical benefits observed in patients with post-operative or post-TAVI regression of LVH suggest that drugs with the highest ability to attenuate LVH in arterial hypertension, i.e. renin-angiotensin system (RAS) blockers [2], would be indicated also for this purpose in AS. In fact, angiotensin-converting enzyme blockers (ACEI) and angiotensin receptor blockers (ARB) exert an additional protective effect against $\mathrm{LVH}$, beyond that resulting from blood pressure lowering $[2,56]$. RAS blockade attenuates both cardiomyocyte hypertrophy and the expansion of extracellular matrix $[2,56]$, and myocardial fibrosis with overexpression of the angiotensin-converting enzyme accompanies severe AS [57]. Weidemann et al. [58] demonstrated that a greater extent of pre-operative myocardial fibrosis was associated with longitudinal LV systolic dysfunction and no clinical improvement after surgery for severe symptomatic AS. On the other hand, according to traditional views, severe AS is perceived as a relative, yet recognized, contraindication to $A C E I$ and $A R B$, owing to the risk of hypotension and coronary hypoperfusion.

Nevertheless, a report from a large Scottish registry of a total of 2,117 patients with AS suggested good tolerance of ACEI or ARB (i.e. no effect on blood pressure), associated with even a lower risk of all-cause mortality and adverse CV events within an about 4-year follow-up, regardless of stenosis severity [59]. It can be speculated that this effect might partially be linked to lower LV mass in patients on ACEI or ARB. In agreement with this concept, in a cross-sectional analysis of 428 subjects with 
severe AS and preserved EF, Goh et al. [60] observed decreased odds of having LVH in those treated with ACEI or ARB with reference to their counterparts receiving neither ACEI nor ARB. The notion of potential benefits of RAS blockade in AS is also consistent with a prospective study by Bang et al. [61], who observed a lower time-dependent increase in LV mass, but no effect on AS progression rate, in $769 \mathrm{ACEl}$ or ARB users over a mean follow-up of 4.3 years of 1,873 patients with mild or moderate AS participating in the SEAS trial. In addition, the incidence of all study end-points, including all-cause death, CV death or sudden cardiac death was similar according to the use of RAS antagonists [61]. Finally, potential clinical benefits of ACEI/ARB in less than severe AS could also be linked to their capability to slow down the progression of renal function decline, recently shown to contribute to the development of excessive LVH in moderate AS, as described by our group [62].

Anyway, safety concerns regarding RAS blockade in severe AS are not more valid after a successful TAVI or surgical aortic valve replacement. Thus, RAS blockade appears to be a promising tool to further potentiate LVH regression after TAVI or aortic valve surgery. Furthermore, the capability of ACEI/ARB to attenuate also myocardial fibrosis [56], presumably less responsive to post-procedural afterload reduction than myofibrillar hypertrophy, is especially promising in post-TAVI patients, because the extent of preoperative-myocardial fibrosis predisposed to the post-operative persistence of residual LVH [38].
In a registry of 1,215 patients undergoing TAVI, Ochiai et al. [63] recently demonstrated 4-fold higher 6-month regression of LV mass ( $9 \%$ vs. $2 \%$ ) and over 2 -fold lower all-cause 2-year mortality in patients treated with ACEI or ARB after the procedure.

Hence, further studies are warranted to optimize pre-interventional and post-interventional strategies aimed at counteracting LV remodeling in AS.

\section{Summary}

Left ventricular hypertrophy has traditionally been perceived as a compensatory mechanism aimed at the maintenance of LV systolic function under chronic LV pressure overload, such as arterial hypertension and AS. Left ventricular hypertrophy development is dependent on afterload-modifying factors, i.e. AS severity and reduced compliance of large systemic arteries, with an additional impact of non-hemodynamic contributors, e.g. metabolic syndrome and chronic kidney disease. Notably, in AS patients, both the presence and magnitude of $\mathrm{LVH}$ are associated with an increased risk of adverse CV events, especially when LV mass is inappropriately high, i.e. disproportionate to LV afterload. Importantly, the negative prognostic effect of excessive LVH or LV concentricity in severe AS was observed irrespective of treatment strategy (medical therapy or interventional approach), being also reported in patients after surgical valve replacement or TAVI, in whom severe pre-operative LVH and its post-operative persistence lessened the clinical

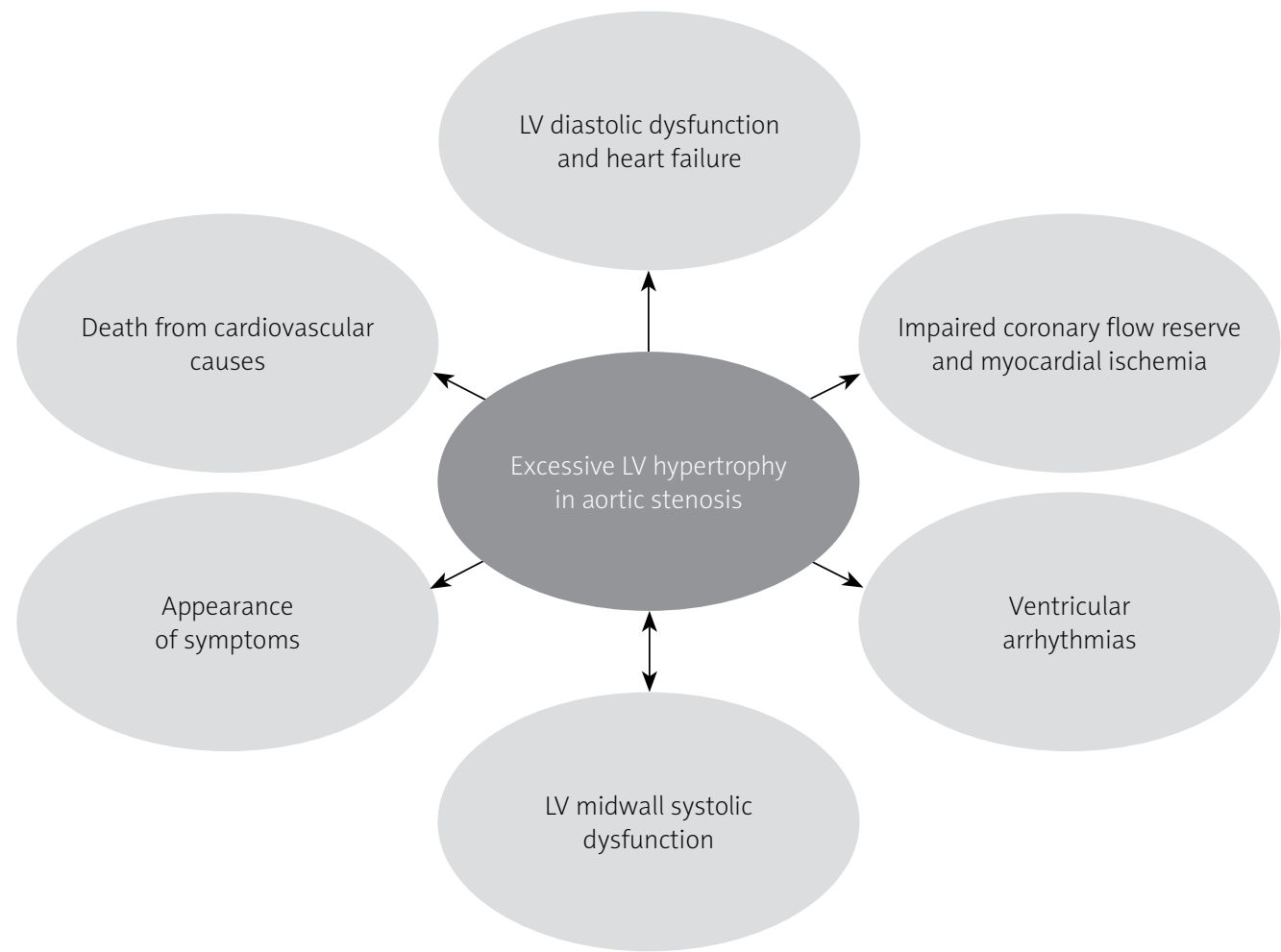

Figure 1. Detrimental effects of excessive left ventricular (LV) hypertrophy, i.e. inappropriately high LV mass disproportionate to LV afterload, in aortic stenosis 
benefits of aortic valve-related interventions. In particular, early LVH regression after TAVI was associated with better LV function recovery and reduced risk of heart failure hospitalizations at 1-year follow-up. Intriguingly, patients with severe AS and inadequate LV mass (i.e. too low) had a similar CV risk to those with an appropriate LV mass relative to LV afterload, despite markedly increased LV wall stress. Thus, theoretical benefits of afterload reduction are presumably offset by detrimental consequences of LVH (Figure 1), including LV diastolic dysfunction, impairment of coronary flow reserve and propensity to ventricular arrhythmias. In addition, subtle LV systolic dysfunction at the midwall level and reduced LV wall stress were described in subjects with AS and inappropriately high LV mass. Accordingly, excessive LVH can also be enhanced by primary myocardial dysfunction, being thereby a likely compensatory, albeit inefficient mechanism whose goal would be to restore LV function via reduced LV wall stress. Irrespective of pathophysiological considerations, drugs with the highest ability to attenuate LVH, ACEI and ARB seem a promising tool in AS, especially after a successful aortic valve replacement, when classical safety concerns regarding RAS blockade in severe AS are no longer present.

\section{Conclusions}

Excessive LVH appears to be a potential additional therapeutic target in AS. Randomized studies are warranted with regard to RAS blockade in AS patients with severe LVH, especially after surgical or transcatheter aortic valve replacement, in order to further optimize longterm prognosis.

\section{Acknowledgments}

Bernadeta Chyrchel and Andrzej Surdacki are joint senior authors on this work (SSG tutors).

\section{Conflict of interest}

The authors declare no conflict of interest.

\section{References}

1. O'Rourke MF, Staessen JA, Vlachopoulos C, et al. Clinical applications of arterial stiffness; definitions and reference values. Am J Hypertens 2002; 15: 426-44.

2. Grodzicki T, Messerli FH. The heart in the hypertensive elderly. J Hum Hypertens 1998; 12: 593-7.

3. De Simone G, Kitzman DW, Palmieri V, et al. Association of inappropriate left ventricular mass with systolic and diastolic dysfunction: the HyperGEN study. Am J Hypertens 2004; 17: 828-33.

4. Palmieri V, de Simone G, Roman MJ, et al. Ambulatory blood pressure and metabolic abnormalities in hypertensive subjects with inappropriately high left ventricular mass. Hypertension 1999; 34: 1032-40.

5. Palmieri V, Wachtell K, Gerdts E, et al. Left ventricular function and hemodynamic features of inappropriate left ventricular hypertrophy in patients with systemic hypertension: the LIFE study. Am Heart J 2001; 141: 784-91.

6. Galderisi M, de Simone G, Cicala S, et al. Coronary flow reserve in hypertensive patients with appropriate or inappropriate left ventricular mass. J Hypertens 2003; 21: 2183-8.

7. De Simone G, Verdecchia P, Pede S, et al. Prognosis of inappropriate left ventricular mass in hypertension: the MAVI Study. Hypertension 2002; 40: 470-6.

8. De Simone G, Gottdiener JS, Chinali M, Maurer MS. Left ventricular mass predicts heart failure not related to previous myocardial infarction: the Cardiovascular Health Study. Eur Heart J 2008; 29: 741-7.

9. Shim CY. Arterial-cardiac interaction: the concept and implications. J Cardiovasc Ultrasound 2011; 19: 62-6.

10. Briand M, Dumesnil JG, Kadem L, et al. Reduced systemic arterial compliance impacts significantly on left ventricular afterload and function in aortic stenosis: implications for diagnosis and treatment. J Am Coll Cardiol 2005; 46: 291-8.

11. Dulgheru R, Magne J, Capoulade R, et al. Impact of global hemodynamic load on exercise capacity in aortic stenosis. Int J Cardiol 2013; 168: 2272-7.

12. Ramamurthi A, Pandian NG, Gangadharamurthy D, et al. The syndrome of degenerative calcific aortic stenosis: prevalence of multiple pathophysiologic disorders in association with valvular stenosis and their implications. Echocardiography 2013; 30: 1-7.

13. Rajani R, Rimington $\mathrm{H}$, Nabeebaccus A, et al. Asymptomatic aortic stenosis: the influence of the systemic vasculature on exercise time. J Am Soc Echocardiogr 2012; 25: 613-9.

14. Kruszelnicka O, Chmiela M, Bobrowska, et al. Depressed systemic arterial compliance is associated with the severity of heart failure symptoms in moderate-to-severe aortic atenosis: a cross-sectional retrospective study. Int J Med Sci 2015; 12: 552-8.

15. Tongue AG, Dumesnil JG, Laforest I, et al. Left ventricular longitudinal shortening in patients with aortic stenosis: relationship with symptomatic status. J Heart Valve Dis 2003; 12: 142-9.

16. Dahl JS, Christensen NL, Videbæk L, et al. Left ventricular diastolic function is associated with symptom status in severe aortic valve stenosis. Circ Cardiovasc Imaging 2014; 7: 142-8.

17. Dalsgaard M, Kjaergaard J, Pecini R, et al. Predictors of exercise capacity and symptoms in severe aortic stenosis. Eur J Echocardiogr 2010; 11: 482-7.

18. Gerdts E, Rossebø AB, Pedersen TR, et al. Relation of left ventricular mass to prognosis in initially asymptomatic mild to moderate aortic valve stenosis. Circ Cardiovasc Imaging 2015; 8: e003644.

19. De Simone G, Devereux RB, Kimball TR, et al. Interaction between body size and cardiac workload: influence on left ventricular mass during body growth and adulthood. Hypertension 1998; 31: 1077-82.

20. Palmieri V, Okin PM, de Simone G, et al. Electrocardiographic characteristics and metabolic risk factors associated with inappropriately high left ventricular mass in patients with electrocardiographic left ventricular hypertrophy: the LIFE Study. J Hypertens 2007; 25: 1079-85.

21. Cioffi G, de Simone G, Cramariuc D, et al. Inappropriately high left-ventricular mass in asymptomatic mild-moderate aortic stenosis. J Hypertens 2012; 30: 421-8.

22. Mureddu GF, Pasanisi F, Palmieri V, et al. Appropriate or inappropriate left ventricular mass in the presence or absence of 
prognostically adverse left ventricular hypertrophy. J Hypertens 2001; 19: 1113-9.

23. Lund BP, Gohlke-Bärwolf C, Cramariuc D, et al. Effect of obesity on left ventricular mass and systolic function in patients with asymptomatic aortic stenosis (a Simvastatin Ezetimibe in Aortic Stenosis [SEAS] substudy). Am J Cardiol 2010; 105: 1456-60.

24. Pagé A, Dumesnil JG, Clavel MA, et al.; ASTRONOMER Investigators. Metabolic syndrome is associated with more pronounced impairment of left ventricle geometry and function in patients with calcific aortic stenosis: a substudy of the ASTRONOMER (Aortic Stenosis Progression Observation Measuring Effects of Rosuvastatin). J Am Coll Cardiol 2010; 55: 1867-74.

25. Aurigemma GP, Silver KH, Priest MA, Gaasch WH. Geometric changes allow normal ejection fraction despite depressed myocardial shortening in hypertensive left ventricular hypertrophy. J Am Coll Cardiol 1995; 26: 195-202.

26. Aurigemma GP, Devereux RB, de Simone G, et al. Myocardial function and geometry in hypertensive subjects with low levels of afterload. Am Heart J 2002; 143: 546-51.

27. Cioffi G, Faggiano P, Vizzardi E, et al. Prognostic effect of inappropriately high left ventricular mass in asymptomatic severe aortic stenosis. Heart 2011; 97: 301-7.

28. Orsinelli DA, Aurigemma GP, Battista S, et al. Left ventricular hypertrophy and mortality after aortic valve replacement for aortic stenosis. A high risk subgroup identified by preoperative relative wall thickness. J Am Coll Cardiol 1993; 22: 1679-83.

29. Duncan Al, Lowe BS, Garcia MJ, et al. Influence of concentric left ventricular remodeling on early mortality after aortic valve replacement. Ann Thorac Surg 2008; 85: 2030-9.

30. Debry N, Maréchaux S, Rusinaru D, et al. Prognostic significance of left ventricular concentric remodelling in patients with aortic stenosis. Arch Cardiovasc Dis 2017; 110: 26-34.

31. Seiler C, Jenni R. Severe aortic stenosis without left ventricular hypertrophy: prevalence, predictors, and short-term follow up after aortic valve replacement. Heart 1996; 76: 250-5.

32. Barasch E, Kahn J, Petillo F, Pollack S, et al. Absence of left ventricular hypertrophy in severe isolated aortic stenosis and preserved left ventricular systolic function. J Heart Valve Dis 2014; 23: 1-8.

33. De Simone G, Palmieri V, Koren MJ, et al. Prognostic implications of the compensatory nature of left ventricular mass in arterial hypertension. J Hypertens 2001; 19: 119-25.

34. Carroll JD, Carroll EP, Feldman T, et al. Sex-associated differences in left ventricular function in aortic stenosis of the elderly. Circulation 1992; 86: 1099-107.

35. Villari B, Campbell SE, Schneider J, et al. Sex-dependent differences in left ventricular function and structure in chronic pressure overload. Eur Heart J 1995; 16: 1410-9.

36. Petrov G, Regitz-Zagrosek V, Lehmkuhl E, et al. Regression of myocardial hypertrophy after aortic valve replacement: faster in women? Circulation 2010; 122 (11 Suppl): S23-8.

37. Petrov G, Dworatzek E, Schulze TM, et al. Maladaptive remodeling is associated with impaired survival in women but not in men after aortic valve replacement. JACC Cardiovasc Imaging 2014; 7: 1073-80.

38. Gavina C, Falcão-Pires I, Pinho P, et al. Relevance of residual left ventricular hypertrophy after surgery for isolated aortic stenosis. Eur J Cardiothorac Surg 2016; 49: 952-9.

39. Sharma UC, Barenbrug P, Pokharel S, et al. Systematic review of the outcome of aortic valve replacement in patients with aortic stenosis. Ann Thorac Surg 2004; 78: 90-5.
40. Ali A, Patel A, Ali Z, et al. Enhanced left ventricular mass regression after aortic valve replacement in patients with aortic stenosis is associated with improved long-term survival. J Thorac Cardiovasc Surg 2011; 142: 285-91.

41. Beach JM, Mihaljevic T, Rajeswaran J, et al. Ventricular hypertrophy and left atrial dilatation persist and are associated with reduced survival after valve replacement for aortic stenosis. J Thorac Cardiovasc Surg 2014; 147: 362-9.

42. Hatani T, Kitai T, Murai R, et al. Associations of residual left ventricular and left atrial remodeling with clinical outcomes in patients after aortic valve replacement for severe aortic stenosis. J Cardiol 2016; 68: 241-7.

43. Une D, Mesana L, Chan V, et al. Clinical impact of changes in left ventricular function after aortic valve replacement: analysis from 3112 patients. Circulation 2015; 132: 741-7.

44. Smith CR, Leon MB, Mack MJ, et al. Transcatheter versus surgical aortic-valve replacement in high-risk patients. N Engl J Med 2011; 364: 2187-98.

45. Hahn RT, Pibarot P, Stewart WJ, et al. Comparison of transcatheter and surgical aortic valve replacement in severe aortic stenosis: a longitudinal study of echocardiography parameters in cohort A of the PARTNER trial (Placement of Aortic Transcatheter Valves). J Am Coll Cardiol 2013; 61: 2514-21.

46. Al-Hawwas M, Marmagkiolis K, Mehta JL. The impact of transcatheter aortic valve implantation and surgical aortic valve replacement on left ventricular remodeling. Am J Cardiol 2017; 120: 1198-202.

47. Douglas PS, Hahn RT, Pibarot P, et al. Hemodynamic outcomes of transcatheter aortic valve replacement and medical management in severe, inoperable aortic stenosis: a longitudinal echocardiographic study of cohort B of the PARTNER trial. J Am Soc Echocardiogr 2015; 28: 210-7.

48. Pibarot P, Weissman NJ, Stewart WJ, et al. Incidence and sequelae of prosthesis-patient mismatch in transcatheter versus surgical valve replacement in high-risk patients with severe aortic stenosis: a PARTNER trial cohort-A analysis. J Am Coll Cardiol 2014; 64: 1323-34.

49. Lindman BR, Stewart WJ, Pibarot P, et al. Early regression of severe left ventricular hypertrophy after transcatheter aortic valve replacement is associated with decreased hospitalizations. JACC Cardiovasc Interv 2014; 7: 662-73.

50. Panoulas VF, Ruparelia N, Franks R, et al. The impact of gender on outcomes following transcatheter aortic valve implantation: true effect or bias? J Heart Valve Dis 2016; 25: 552-6.

51. Williams M, Kodali SK, Hahn RT, et al. Sex-related differences in outcomes after transcatheter or surgical aortic valve replacement in patients with severe aortic stenosis: insights from the PARTNER Trial (Placement of Aortic Transcatheter Valve). J Am Coll Cardiol 2014; 63: 1522-8

52. Rymuza B, Zbroński K, Ścisło P, et al. Left ventricular remodelling pattern and its relation to clinical outcomes in patients with severe aortic stenosis treated with transcatheter aortic valve implantation. Adv Interv Cardiol 2017; 13: 288-94.

53. Tzikas A, Geleijnse ML, Van Mieghem NM, et al. Left ventricular mass regression one year after transcatheter aortic valve implantation. Ann Thorac Surg 2011; 91: 685-91.

54. Nucifora G, Tantiongco JP, Crouch G, et al. Changes of left ventricular mechanics after trans-catheter aortic valve implantation and surgical aortic valve replacement for severe aortic stenosis: a tissue-tracking cardiac magnetic resonance study. Int J Cardiol 2017; 228: 184-90.

55. Costantino MF, Galderisi M, Dores E, et al. Parallel improvement of left ventricular geometry and filling pressure after transcath- 
eter aortic valve implantation in high risk aortic stenosis: comparison with major prosthetic surgery by standard echo Doppler evaluation. Cardiovasc Ultrasound 2013; 11: 18.

56. Kjeldsen SE, Strand A, Julius S, Okin PM. Mechanism of angiotensin II type 1 receptor blocker action in the regression of left ventricular hypertrophy. J Clin Hypertens (Greenwich) 2006; 8: 487-92.

57. Fielitz J, Hein S, Mitrovic V, et al. Activation of the cardiac renin-angiotensin system and increased myocardial collagen expression in human aortic valve disease. J Am Coll Cardiol 2001; 37: 1443-9.

58. Weidemann F, Herrmann S, Störk S, et al. Impact of myocardial fibrosis in patients with symptomatic severe aortic stenosis. Circulation 2009; 120: 577-84.

59. Nadir MA, Wei L, Elder DH, et al. Impact of renin-angiotensin system blockade therapy on outcome in aortic stenosis. J Am Coll Cardiol 2011; 58: 570-6.

60. Goh SS, Sia CH, Ngiam NJ, et al. Effect of renin-angiotensin blockers on left ventricular remodeling in severe aortic stenosis. Am J Cardiol 2017; 119: 1839-45.

61. Bang CN, Greve AM, Køber L, et al. Renin-angiotensin system inhibition is not associated with increased sudden cardiac death, cardiovascular mortality or all-cause mortality in patients with aortic stenosis. Int J Cardiol 2014; 175: 492-8.

62. Dlugosz D, Bolt K, Sam WS, et al. Excessive left ventricular hypertrophy in moderate degenerative aortic stenosis: an ineffective compensatory mechanism triggered by primary myocardial dysfunction and enhanced by concomitant mild renal impairment? Kardiol Pol 2018 (in press).

63. Ochiai T, Saito S, Yamanaka F, et al. Renin-angiotensin system blockade therapy after transcatheter aortic valve implantation. Heart 2018; 104: 644-51. 